\title{
Vorwort zur ersten Auflage
}

Die Numerische Mathematik beschäftigt sich mit der Entwicklung, Analyse, Implementierung und Testung von numerischen Rechenverfahren, die für die Lösung mathematischer Problemstellungen auf einem Computer geeignet sind. Im Vordergrund steht dabei nicht die Diskussion von Existenz und Eindeutigkeit der entsprechenden Lösungen, sondern deren konkrete Berechnung in Form von Maschinenzahlen. Die Existenz mindestens einer Lösung wird deshalb stets als nachgewiesen vorausgesetzt. Im allgemeinen erhält man als Ergebnis einer numerischen Rechnung nur eine Approximation für die (unbekannte) exakte Lösung. Diese Näherung kann durch die Anhäufung der unvermeidbaren Rundungsfehler völlig verfälscht sein, da auf einem Computer nicht der Körper der reellen Zahlen, sondern nur eine endliche Zahlenmenge, die sogenannten Maschinenzahlen, zur Verfügung steht. Somit gehören zu einer numerischen Rechnung auch stets Fehlerabschätzungen. Unterschiedlich genaue Näherungen für ein und dieselbe exakte Lösung werden als qualitativ gleichwertig angesehen, wenn deren Fehler durch die Erhöhung des technischen Aufwandes beliebig klein gemacht werden können.

Durch die gegenwärtige Entwicklung extrem schneller Rechner mit großen Speichermedien und neuer leistungsfähiger mathematischer Methoden wird es zunehmend möglich, mathematische Problemstellungen einer Lösung zuzuführen, denen immer komplexere und realitätsnähere Modelle aus den konkreten Anwendungen zugrundeliegen. Heute ist man bereits in der Lage, durch numerische Simulationen auf dem Rechner ganze technische Abläufe vor der eigentlichen Fertigung zu verstehen und zu beherrschen. Dies trifft auch auf die sehr umfangreichen und extrem kostenaufwendigen Experimente in den Naturwissenschaften zu. An der Nahtstelle zwischen Numerischer Mathematik, Informatik sowie den Natur- und Ingenieurwissenschaften hat sich bereits eine neue Wissenschaftsdisziplin entwickelt, das Wissenschaftliche Rechnen (Scientific Computing). Wichtige Komponenten dieser neuen Disziplin müssen deshalb in der Ausbildung auf dem Gebiet der Numerischen Mathematik berücksichtigt werden.

Das vorliegende Lehrbuch über die Grundlagen der Numerischen Mathematik ist aus Manuskripten zu Vorlesungen und Seminaren, die der Verfasser seit etwa 15 Jahren an der Friedrich-Schiller-Universität Jena abgehalten hat, hervorgegangen. Es richtet sich an Studierende der Mathematik (einschließlich Lehramt für Gymnasien), Informatik und Physik. Die Themen wurden so ausgewählt, dass anhand dieses Buches der für die deutschen Universitäten typische Grundkurs „Numerische Mathematik“ studiert werden kann. Dieser findet je nach Universitätsprofil und Studiengang im 2. bis 4. Semester statt. Die numerische Behandlung gewöhnlicher und partieller Differentialgleichungen bleibt deshalb unberücksichtigt. Der Text sollte in den Grundzügen schon mit geringen Vorkenntnissen der Linearen Algebra und Analysis verständlich sein. 
Schwerpunktmäßig werden im Buch diejenigen numerischen Techniken betrachtet, die auf den heute üblichen Computern in Form von Software-Paketen implementiert vorliegen und in den Anwendungen tatsächlich auch zum Einsatz kommen. Eine häufig genutzte Bibliothek für numerische Software ist die Netlib, die im World Wide Web unter der URL bzw. E-mail Adresse http://www.netlib.org bzw. netlib@netlib.org zu erreichen ist. Um die Studenten schon sehr schnell mit der numerischen Software vertraut zu machen, wird an der Friedrich-Schiller-Universität Jena das Software-Paket MATLAB ${ }^{\circledR 1}$ im Grundkurs zur Numerischen Mathematik eingesetzt. Im vorliegenden Buch sind die angegebenen numerischen Algorithmen in einem einfachen Pseudocode formuliert, so dass ihre Implementierung in den Sprachelementen von MATLAB ${ }^{\circledR}$ einfach zu realisieren ist, darüber hinaus aber auch andere moderne Programmiersprachen Verwendung finden können. Es gehört zu den Zielen des Verfassers, neben den theoretischen Grundlagen auch die experimentelle Seite der Numerischen Mathematik zur Geltung zu bringen. Numerische Demonstrationen unter Verwendung moderner Multimedia-Techniken auf einem Computer zeigen dem Studierenden die vielfältigen Steuerungsmöglichkeiten der numerischen Algorithmen besonders einprägsam auf. Sie führen gleichzeitig in die experimentelle Numerik ein, die die Grundlage der neuen Wissenschaftsdisziplin Wissenschaftliches Rechnen ist.

Am Ende eines jeden Kapitels sind Aufgaben zur Übung der gewonnenen theoretischen und praktischen Fertigkeiten angegeben. Auch hier wird häufig der Bezug zur MATLAB ${ }^{\circledR}$ hergestellt. Für weitergehende interessante Übungsaufgaben, auch zur Vorbereitung auf die (leider oftmals nicht zu umgehenden) Prüfungen, sei auf die im gleichen Verlag erschienene zweibändige Aufgabensammlung von N. Herrmann [49] verwiesen.

Meinem Kollegen, Herrn Dr. Dieter Kaiser möchte ich für die Hilfe bei der Erstellung der Abbildungen meinen Dank aussprechen. Gleichfalls möchte ich allen meinen Übungsassistenten für gelegentliche wertvolle Hinweise sowie dem Verlag für die Unterstützung bei der Herausgabe des Lehrbuches danken.

Jena, im Juli 2000

Martin Hermann

$\overline{1 \text { MATLAB }}{ }^{\circledR}$ ist eine registrierte Handelsmarke der Firma The MathWorks Inc., Natick, MA, U.S.A. 